\title{
Treatment of thoracolumbar trauma by short-segment percutaneous transpedicular screw instrumentation: prospective comparative study with a minimum 2-year follow-up
}

\section{Clinical article}

\author{
Petr Vanek, M.D., ${ }^{1}$ Ondrej Bradac, M.D., M.Sc., ${ }^{1}$ Renata Konopkova, Ph.D., ${ }^{2}$ \\ Patricia de Lacy, M.D., ${ }^{3}$ Jiri Lacman, M.D., ${ }^{4}$ and Vladimir Benes, M.D., Ph.D. ${ }^{1}$
}

${ }^{1}$ Department of Neurosurgery, Charles University, 1st Faculty of Medicine, Military University Hospital, Prague; ${ }^{2}$ Department of Anatomy and Biomechanics, Faculty of Physical Education and Sport, Charles University, Prague, Czech Republic; ${ }^{3}$ Department of Neurosurgery, Royal Hallamshire Hospital, Sheffield, United Kingdom; and ${ }^{4}$ Department of Radiology, Military University Hospital, Prague, Czech Republic

Object. The main aim of this study was to compare clinical and radiological outcomes after stabilization by a percutaneous transpedicular system and stabilization from the standard open approach for thoracolumbar spine injury.

Methods. Thirty-seven consecutive patients were enrolled in the study over a period of 16 months. Patients were included in the study if they experienced 1 thoracolumbar fracture (A3.1-A3.3, according to the AO/Magerl classification), had an absence of neurological deficits, had no other significant injuries, and were willing to participate. Eighteen patients were treated by short-segment, minimally invasive, percutaneous pedicle screw instrumentation. The control group was composed of 19 patients who were stabilized using a short-segment transpedicular construct, which was performed through a standard midline incision. The pain profile was assessed by a visual analog scale (VAS), and overall satisfaction by a simple 4-stage scale relating to performance of daily activities. Working ability and return to original occupation were also monitored. Radiographic follow-up was defined by the vertebral body index (VBI), vertebral body angle (VBA), and bisegmental Cobb angle. The accuracy of screw placement was examined using CT.

Results. The mean surgical duration in the percutaneous screw group was $53 \pm 10$ minutes, compared with $60 \pm 9$ minutes in the control group ( $\mathrm{p}=0.032)$. The percutaneous screw group had a significantly lower perioperative blood loss of $56 \pm 17 \mathrm{ml}$, compared with $331 \pm 149 \mathrm{ml}$ in the control group $(\mathrm{p}<0.001)$. Scores on the VAS in patients in the percutaneous screw group during the first 7 postoperative days were significantly lower than those in the control group $(\mathrm{p}<0.001)$. There was no significant difference between groups in VBI, VBA, and Cobb angle values during follow-up. There was no significant difference in screw placement accuracy between the groups and no patients required surgical revision. There was no significant difference between groups in overall satisfaction at the 2-year follow-up $(\mathrm{p}=0.402)$. Working ability was insignificantly better in the percutaneous screw group; previous working position was achieved in 17 patients in this group and in 12 cases in the control group $(p=0.088)$.

Conclusions. This study confirms that the percutaneous transpedicular screw technique represents a viable option in the treatment of preselected thoracolumbar fractures. A significant reduction in blood loss, postoperative pain, and surgical time were the main advantages associated with this minimally invasive technique. Clinical, functional, and radiological results were at least the same as those achieved using the open technique after a 2-year follow-up. The short-term benefits of the percutaneous transpedicular screw technique are apparent, and long-term results have to be studied in other well-designed studies evaluating the theoretical benefit of the percutaneous technique and assessing whether the results of the latter are as durable as the ones achieved by open surgery. (http://thejns.org/doi/abs/10.3171/2013.11 .SPINE13479)

$\begin{array}{llllll}\text { KEY WoRdS } & \bullet & \text { thoracolumbar } \\ \text { stabilization } & \bullet & \text { outcome }\end{array}$

$\mathrm{T}$ HE generally accepted, current surgical management of thoracolumbar trauma involves open exposure, placement of spinal instrumentation, cor-

Abbreviations used in this paper: VAS = visual analog scale; $\mathrm{VBA}=$ vertebral body angle; $\mathrm{VBI}=$ vertebral body index. rection of posttraumatic deformity, and fusion. In cases in which there is significant spinal canal compromise, decompression of the neural structures is usually performed.

This article contains some figures that are displayed in color online but in black-and-white in the print edition. 


\section{Thoracolumbar trauma and percutaneous transpedicular fixation}

There are two main goals of this treatment: immediate stabilization of the affected traumatized segments, and securing conditions so that vertebral healing or segmental fusion occurs. Although the entire philosophy of such treatment can vary from one hospital to another, the most common practice is stabilization using transpedicular screws, which is preceded by subperiosteal muscle separation in the posterior midline approach. These conventional open procedures can be associated with significant perioperative morbidity such as blood loss and/or infection. Verlaan et al. ${ }^{19}$ reviewed the literature and reported a mean blood loss of greater than $1 \mathrm{~L}$ for the posterior, anterior, or combined surgical approaches. According to the analysis performed using data from 138 publications, the average incidence of infectious complications might affect up to $3.1 \%$ of patients in connection with the posterior approach. ${ }^{19}$ Some reports show an incidence of infectious complications as high as $10 \%$ in such groups of patients. ${ }^{15}$ In addition, conventional open procedures can also be associated with approach-related morbidity. Posterior midline approaches have been shown to cause significant postoperative muscle atrophy and scarring as a result of the muscle separation lateral to the facet joints, direct trauma to the vasculature, and increased intramuscular pressure after insertion of commonly used retractors. All these maneuvers cause denervation and ischemia of the paraspinal muscles..$^{711}$

In an effort to minimize soft tissue injury, systems have been developed to place transpedicular screws percutaneously. These systems were originally constructed for the treatment of degenerative conditions of the lumbar spine and such techniques have been available in clinical practice for more than 10 years now. ${ }^{3,8}$ Although surgery using minimally invasive percutaneous fixators leads to the reduction of blood loss and postoperative pain, and a faster return to previous activities, ${ }^{14,18}$ the expectation regarding better long-term outcome has not been confirmed by highly graded scientific studies. In spite of this fact, minimally invasive percutaneous transpedicular screw techniques have already been used within the different fields of spinal surgery, such as surgery of thoracolumbar fractures. The main aim of this study was to compare clinical and radiological outcomes for thoracolumbar spine injury after stabilization by a percutaneous transpedicular screw system, and stabilization using the standard open approach.

\section{Methods}

\section{Study Population}

Thirty-seven consecutive patients were enrolled in this prospective study over a period of 16 months. All patients were urgently admitted to the Military University Hospital in Prague following thoracolumbar spine trauma. There were 4 inclusion criteria: 1) 1 thoracolumbar fracture classified as A3.1-A3.3 according to the AO/ Magerl classification (incomplete burst, burst-split, and complete burst fractures) ${ }^{10}$; 2) absence of neurological deficits; 3) no other significant injuries; and 4) willingness to participate in the study. After an explanation of the surgical options, patients signed an informed consent form authorized by the local ethics committee.

\section{Operative Procedures}

All operations were performed within 24 hours of admission, and in the vast majority of cases within 6 hours. Eighteen patients were treated by short-segment (1 level above and 1 level below the fractured vertebra) minimally invasive, percutaneous pedicle screw instrumentation (Sextant, Medtronic; Fig. 1). Screws were placed under control of biplanar fluoroscopy using 2 perpendicular $\mathrm{C}$-arms.

The control group was composed of 19 patients who were stabilized using a short-segment transpedicular construct that was performed through a standard midline incision involving subperiosteal muscle separation (USS, Synthes, or $\mathrm{S}^{4}$, BBraun; Fig. 2). Screw placement was controlled using lateral fluoroscopy and subsequently using final anteroposterior projection after all screws were inserted. There was no fusion performed in the percutaneous group, unlike the control group, in which a posterolateral fusion was performed using local bone mixed with bone substitute. These two described techniques were regularly alternated between each consecutive patient. Therefore, each consecutive patient received a different treatment from the previous one. None of the patients decided to reject the offered procedure. All surgeries were performed by fully qualified spine surgeons with at least 10 years of experience in spine surgery.

\section{Imaging Evaluation}

Radiographic evaluation consisted of supine admission radiography, CT, and MRI. The accuracy of screw placement was examined using CT on the 1st postoperative day and then using standing radiographs obtained after mobilization of each patient. Standing radiographs were again acquired during the entire follow-up period (1, 2, 3, 6, 12 , and 24 months after surgery). Kyphotic deformity and radiographic follow-up were defined by the vertebral body index (VBI: relation between the anterior and posterior wall height of the fractured vertebra), vertebral body angle (VBA: angle defined by the upper and lower endplate of the fractured vertebra), and bisegmental Cobb angle (angle defined by the upper endplate of the first vertebra above the fractured one and by the lower endplate of the first vertebra below the fractured one). When assessing pedicle screw placement, we paid special attention to medial suboptimal screw position, defined as medial pedicle wall breach. The instrumentation was removed from the mobile part of the lumbar spine (below L-2) at 1 to 1.5 years after the surgery.

\section{Postoperative Procedures}

Patients were mobilized on the 1 st or 2 nd day after surgery without any external support and additional physiotherapy was initiated. The primary parts of the subsequent physiotherapy regimen were isometric contractions, training of postural stability, and muscle group harmonization for optimal posture.

The postprocedural global pain profile was assessed using a visual analog scale (VAS) ${ }^{17}$ completed every day by the patients during the 1st week after surgery. Two years after the surgery, we evaluated overall satisfaction and pain by a simple 4-stage scale relating to their performance of daily activities: 1 = without any inconvenience; 

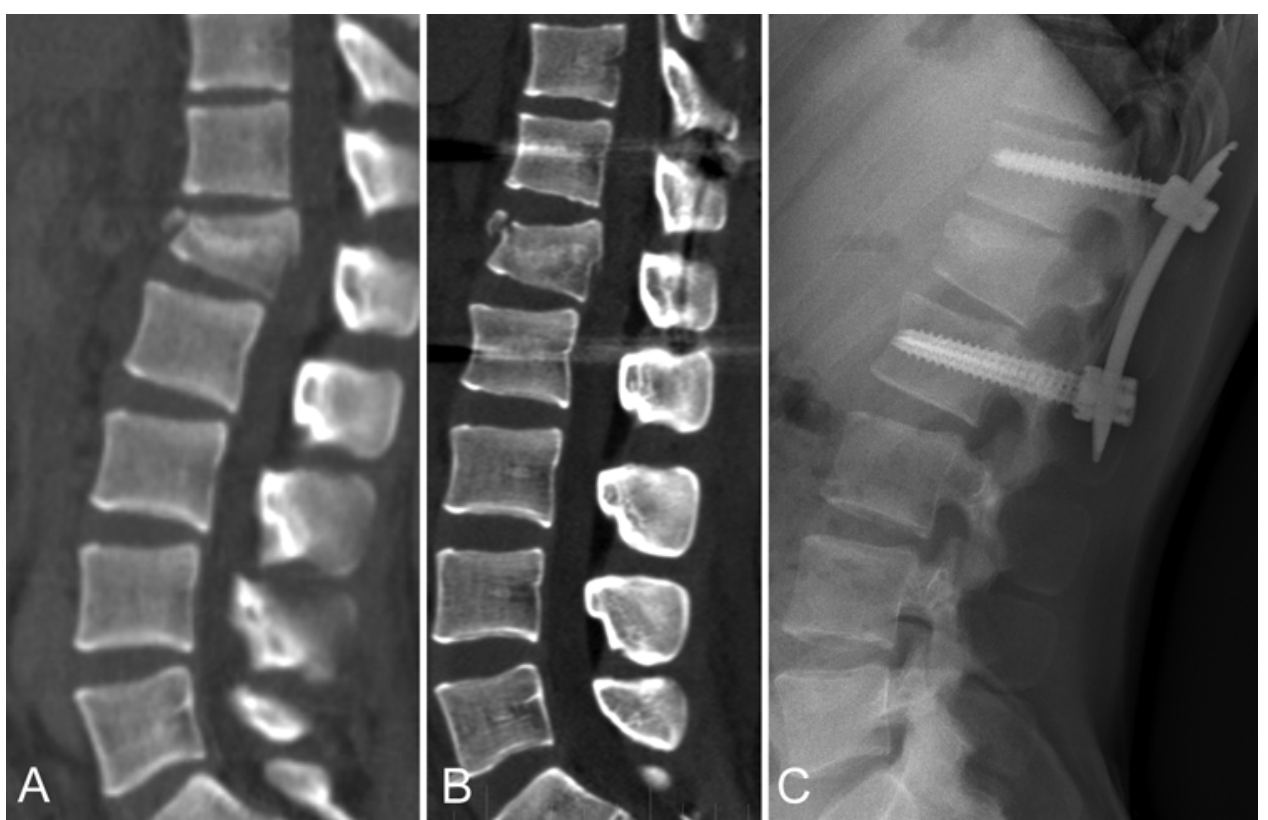

FIG. 1. Sagittal images showing an L-1 burst-split fracture (AO/Magerl classification A3.2) and its repair using percutaneous pedicle screw instrumentation in a 20 -year-old male patient. A: Computed tomography reconstruction obtained prior to surgery. B: Computed tomography reconstruction showing early postoperative control on the 1st day after surgery. C: Plain radiograph obtained at the 2-year follow-up.

$2=$ some residual difficulties, no need of analgesics; 3 $=$ difficulties partially limiting common daily activities, analgesics occasionally; and $4=$ significant limitation during daily activities, suboptimal response on analgesics. The working ability of the patients and their return to their original occupation was also monitored during the first 2 years after the trauma. Functional and radiographic outcome and working ability were assessed by 2 individuals (R.K. and J.L.) who never took part in the previous diagnostic and therapeutic processes.

\section{Statistical Analysis}

Results were evaluated using univariate ANOVA for repeated measures on variables under consideration with post hoc least significant difference testing. The main effects of time and method of treatment, and their interaction, were studied. A comparison of continuous variables was performed using the t-test. A comparison of categorical variables was performed using the chi-square test. All $\mathrm{p}$ values $<0.05$ were considered statistically significant. The STATISTICA software program (version 10.0, StatSoft) was used for statistical evaluation.

\section{Results}

Thirty-five of 37 patients completed follow-up: 18 in the percutaneous group and 17 in the control group. Baseline characteristics, fracture types, and mechanisms of in-
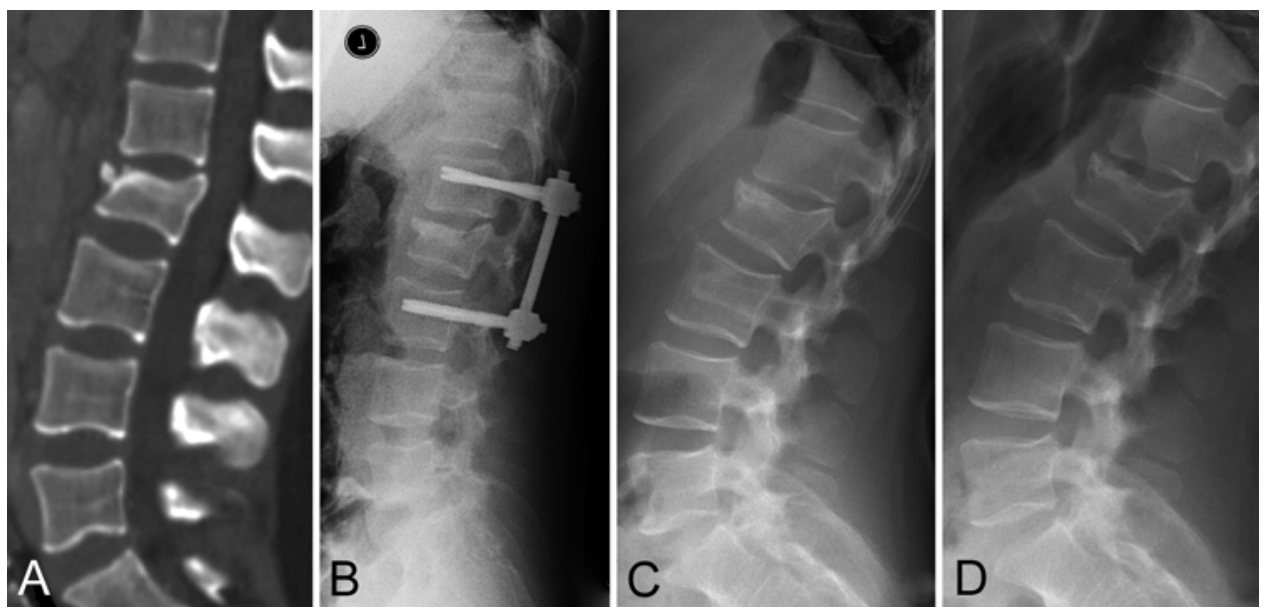

FIG. 2. Sagittal images showing an L-2 burst-split fracture (AO/Magerl classification A3.2) and its repair using a short-segment transpedicular construct in a 24-year-old female patient. A: Computed tomography reconstruction obtained prior to surgery. B: Plain radiograph at the 1-year follow-up, prior to hardware removal. C: Plain radiograph obtained 3 months after hardware removal. D: Plain radiograph at the 2-year follow-up. 


\section{Thoracolumbar trauma and percutaneous transpedicular fixation}

juries of patients in both groups are shown in Tables 1 and 2 . There were no neurological or medical complications recorded in connection with treatment. Only 1 patient from the control group developed a superficial incisional wound infection.

In the VBI parameter we found a significant effect of time (operation, $\mathrm{p}<0.001$ ), but no group effect or group and time interaction. During postoperative follow-up the VBI values were almost identical in both groups (Fig. $3 A)$. The VBA parameter on follow-up showed a significant time effect $(\mathrm{p}<0.001)$ and a group and time interaction $(\mathrm{p}=0.043)$. During the postoperative course VBA values in the control group were slightly lower, but these values were not found to be significantly different at any follow-up evaluation (Fig. 3B). Similar to the VBI and VBA parameters, the Cobb angles showed a significant time effect ( $p<0.001)$, but no group or group and time interaction during the follow-up period (Fig. 3C).

When we assessed total surgical time, the percutaneous treatment group fared slightly but significantly better. The mean surgical duration in the percutaneous group was $53 \pm 10$ minutes, compared with $60 \pm 9$ minutes in the control group $(\mathrm{p}=0.032)$. In comparing perioperative blood loss, the percutaneous treatment group lost an average of only $56 \pm 17 \mathrm{ml}$, compared with an average of 331 $\pm 149 \mathrm{ml}$ in the control group ( $\mathrm{p}<0.001)$.

The pain profile during the first 7 postoperative days, as reflected by VAS scores, is shown in Fig. 3D. A significant effect of time $(p<0.001)$ and group $(p<0.001)$ were found. Patients in the percutaneous treatment group scored significantly lower than those in the control group, and the pain profile showed a similar decline in pain in both groups during the first 7 postoperative days. Overall satisfaction at 2 years after surgery in both groups is shown Table 3. For the sake of statistical evaluation, the 4-level scale was contracted into 2 levels (patients without any inconvenience vs patients with any degree of difficulties). Using this dichotomized approach, no significant difference was found between the groups (15 satisfied patients in the minimally invasive treatment group vs 13 in the control group; $\mathrm{p}=0.402$ ).

Working ability was assessed as the capacity of the patients to return to their previous working position. In the minimally invasive group, this was achieved in 17 patients, and in 12 cases in the control group $(p=0.088)$. A comparison between those patients in whom there were pedicle screws placed too medially on postoperative imaging showed no significant difference between groups ( 2 of 72 in the minimally invasive treatment group vs 5 of 68 in the control group; $p=0.265$ ). Nevertheless, none of the patients required surgical revision. There was no construct failure recorded during follow-up.

\section{Discussion}

In the past decade, there has been an apparent trend to minimize soft tissue injury during spinal surgery. Percutaneous transpedicular screws are currently used in the treatment of thoracolumbar fractures, ${ }^{2,16}$ although there is a substantial lack of scientific evidence for this technique. This minimally invasive approach might involve some important drawbacks. The most often cited drawbacks include a reduced angular stability of the polyaxial screws, lesser potential to correct kyphotic deformity, suboptimal condition for spinal fusion, and the fact that the majority of such systems can provide only short-segment fixation. Although the initial results with short-segment fixation in thoracolumbar trauma cases were dismal, showing mechanical failure in as many as $50 \%$ of patients, ${ }^{12}$ currently the general consensus about the suitability of this therapeutic approach is that it is acceptable in preselected patient groups. ${ }^{23}$ Furthermore, this procedure can even be performed with no intersegmental fusion in patients with supposedly favorable bone healing potential and the absence of significant ligamentous instability. ${ }^{22}$ Therefore, unstable burst-type fractures (AO/Magerl Type A3 classification) without severe kyphotic deformity were selected for this trial.

Assaker ${ }^{1}$ first reported on the application of percutaneous transpedicular fixation in thoracolumbar trauma. He presented results of both a primary, stand-alone technique, or in combination with an anterior endoscopic approach. The average loss of correction was $7.5^{\circ}$, there was no reported construct failure nor loosening, and no difference in long-term outcomes were shown in his case series. ${ }^{1}$ There were only 2 retrospective cohort studies published on this topic until 2012. Wild et al. ${ }^{21}$ retro-

TABLE 1: Baseline characteristics of the patient cohort

\begin{tabular}{lccc}
\hline \multicolumn{1}{c}{ Variable } & Percutaneous Group & Control Group & p Value \\
\hline no. of patients & 18 & 17 & \\
mean age \pm SD & $39.4 \pm 16.9$ & $45.6 \pm 15.3$ & 0.264 \\
no. of males & 14 & 14 & 1.000 \\
profession & & & 1.000 \\
$\quad$ manual worker & 11 & 9 & \\
$\quad$ office worker & 7 & 8 & 0.738 \\
thoracic fracture & 11 & 9 & \\
mean preop values \pm SD & & & 0.848 \\
VBI & $0.67 \pm 0.12$ & $0.68 \pm 0.12$ & 0.635 \\
VBA $\left(^{\circ}\right)$ & $-14.4 \pm 6.5$ & $-13.5 \pm 5.5$ & 0.766 \\
Cobb angle $\left(^{\circ}\right)$ & $-9.3 \pm 10.1$ & $-8.2 \pm 10.5$ & \\
\hline
\end{tabular}


TABLE 2: Fracture characteristics and mechanisms of injuries

\begin{tabular}{lcc}
\hline \multicolumn{1}{c}{ Variable } & Percutaneous Group & Control Group \\
\hline fracture level & & \\
T-9 & 1 & 0 \\
T-12 & 6 & 8 \\
L-1 & 8 & 6 \\
L-2 & 0 & 1 \\
L-3 & 1 & 2 \\
L-4 & 2 & 0 \\
fracture type (AO/Magerl) & & \\
A3.1 & 1 & 1 \\
A3.2 & 6 & 4 \\
A3.3 & 11 & 12 \\
mechanism of injury & & 3 \\
motor vehicle accident & 3 & 13 \\
fall & 10 & 1 \\
sports injury & 5 & \\
\hline
\end{tabular}

spectively reviewed the records of 21 patients who were treated using posterior fixation (mainly for thoracolumbar fracture A3, according to the AO/Magerl classification), without any anterior or posterior fusion, in which the percutaneous system was used in 10 patients and a conventional open procedure in 11 patients. There was no significant difference in the degree of intraoperative reduction or in loss of correction during follow-up at 5 years after implant removal, but the patients in the control group were significantly older and had significantly greater kyphotic deformity. ${ }^{21}$ In 2010 , Wang et al..$^{20}$ published records of 38 patients in a similarly designed trial (17 patients in the percutaneous group and 21 in the open group), showing no significant difference in age or kyphotic deformity between groups. After a mean follow-up of 11.6 months they reported the same radiological results as Wild and colleagues had previously. ${ }^{20}$ Finally, in 2012, the first randomized trial was published comparing the efficacy and safety of 2 paraspinal muscle-sparing surgical approaches in the management of neurologically intact patients after thoracolumbar burst fracture. There were 31 patients in the percutaneous treatment group and 30 in the paraspinal approach group. After more than 3 years, the authors concluded that the paraspinal approach is able to correct deformity in the case of unsuccessful postural reduction, but there were no significant differences between groups in long-term stability. ${ }^{6}$

Radiological data in our study did not deviate from previously published patient series. We found significant correction in all monitored parameters in both groups, and the percutaneous technique was even slightly better compared with the control group in terms of restoration of VBI. During the entire follow-up period there was no significant deterioration in any of the monitored parameters, and the mean loss of correction did not exceed $5^{\circ}$ in either group. According to these data, we concluded that the reduced angular stability of polyaxial screws does not hamper their constructs, compared to the angle-stable fixations in the preselected fracture pattern. Even the correcting capacity appeared to be adequate for A3-type fractures, according to AO/Magerl classification.

Some authors advocate polymethylmethacrylate augmentation of percutaneous transpedicular screws with or without kyphoplasty of fractured vertebrae in patients without osteoporosis. ${ }^{13}$ We do not find this concept to be useful in patients with normal bone supply because screw anchoring appeared to be adequate and sufficient, according to our results. In our opinion, polymethylmethacrylate augmentation only increases the potential risk of perioperative complications. Furthermore, there are no published data to clarify the potential long-term consequences of bone cement placed into the vertebral body, and speculation about interference with the natural vertebral body healing process might be correct. ${ }^{9}$

A wide variation in the rate of transpedicular screw misplacement with the open technique has been reported. Gelalis et al. ${ }^{4}$ analyzed 26 prospective clinical studies including a total of 1105 patients (6617 screws inserted). The percentage of the screws that were fully contained within the pedicles ranged from $69 \%$ to $94 \%$ using the freehand technique. Heintel et al. ${ }^{5}$ recently published results of 502 percutaneously inserted transpedicular screws in 111 patients. All screws were inserted with the assistance of 2-plane conventional fluoroscopy, $98 \%$ were found to have good or excellent position, and only 1 screw had to be revised due to a medial position with a neurological deficit. ${ }^{5}$ We did not reach such a high accuracy as Heintel et al. did, even though our results reached the accuracy of other published studies of the open technique. Additionally, there was no significant difference between the studied groups in our trial and there was also no need for screw revision for a neurological deficit or any suspicion of inadequate fixation strength. Accordingly, the safety and accuracy of transpedicular screw placement is the same for open and percutaneous techniques, and again this is consistent both with our data and published data.

The significantly lower blood loss in the percutaneous group was an expected and unsurprising result. The same finding was published in the vast majority of studies in the literature on this topic. The operative time was significantly reduced in the percutaneous technique compared with the open technique, and this fact was also a very important result for us. Percutaneous transpedicular screw insertion is sometimes considered to be a more technically demanding and time-consuming technique in comparison with the standard open technique. ${ }^{13,18}$ We do not agree with such a statement, in spite of the fact that this technique requires certain experience. Once such a learning curve is overcome and this technique is mastered, the opposite is true.

Postoperative incisional pain was significantly reduced by the percutaneous technique in our study, but the 2-year overall satisfaction and working ability were not affected by either technique, according to our data. The same conclusion was found in every previously performed study. The more interesting finding was that working ability was higher in the percutaneous group; borderline significance was found in favor of this technique. On the other hand, we have to remind ourselves of 
A

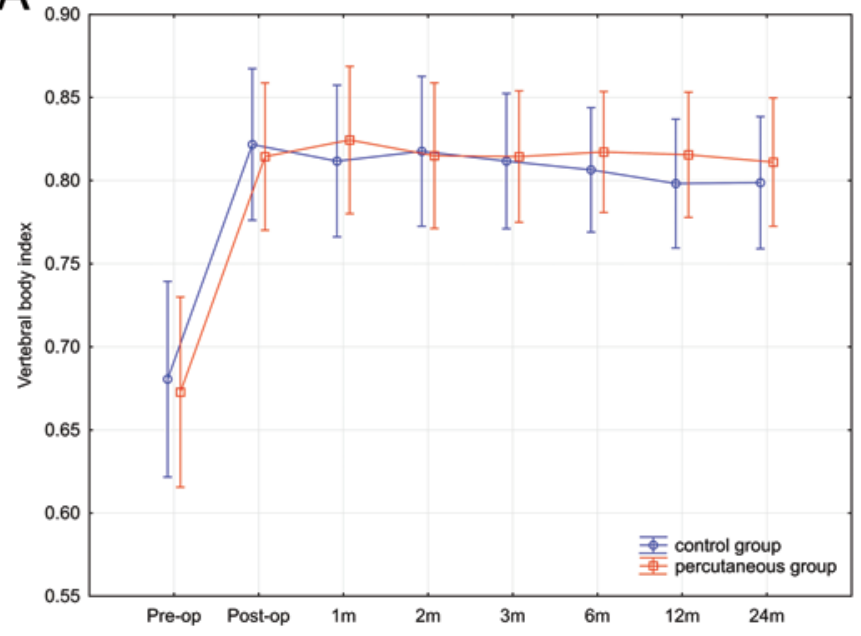

C

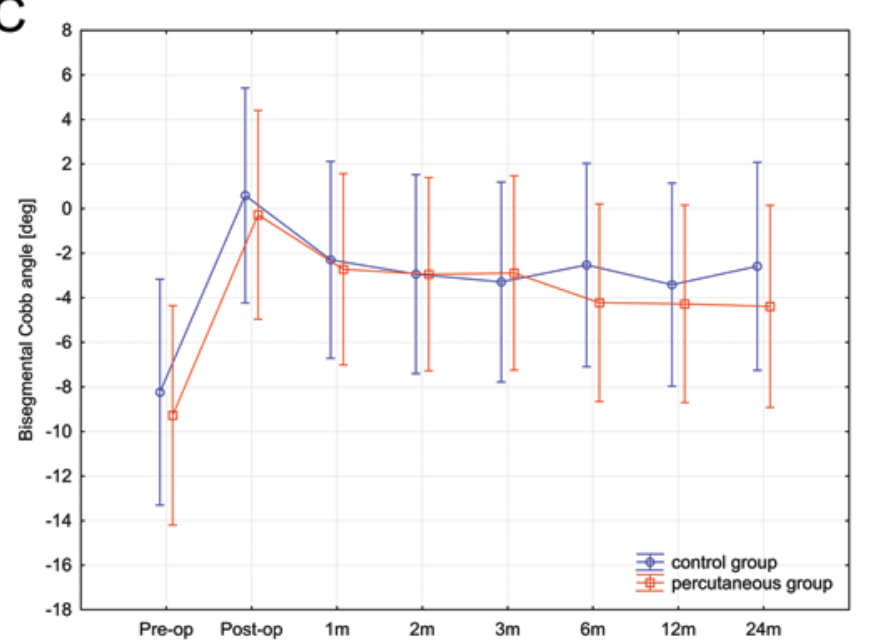

B

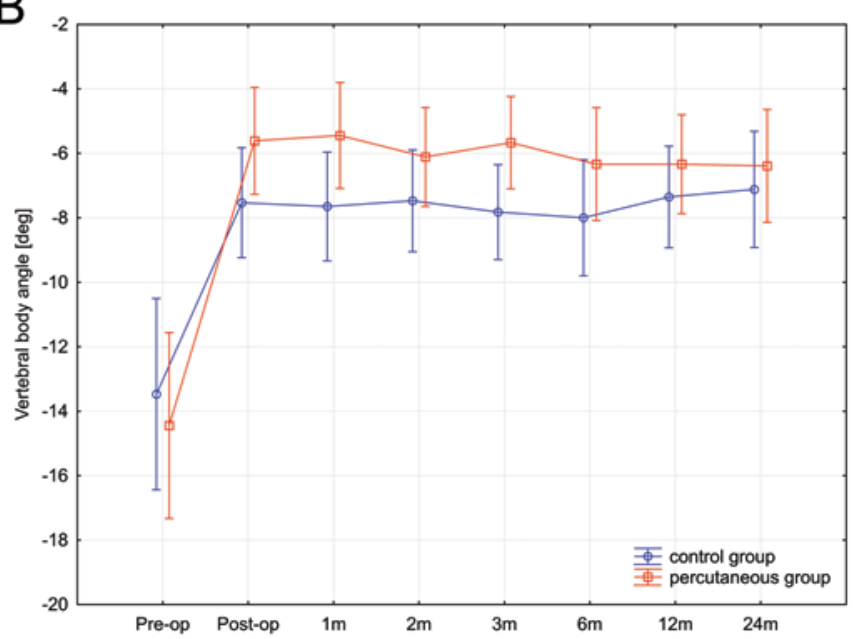

D

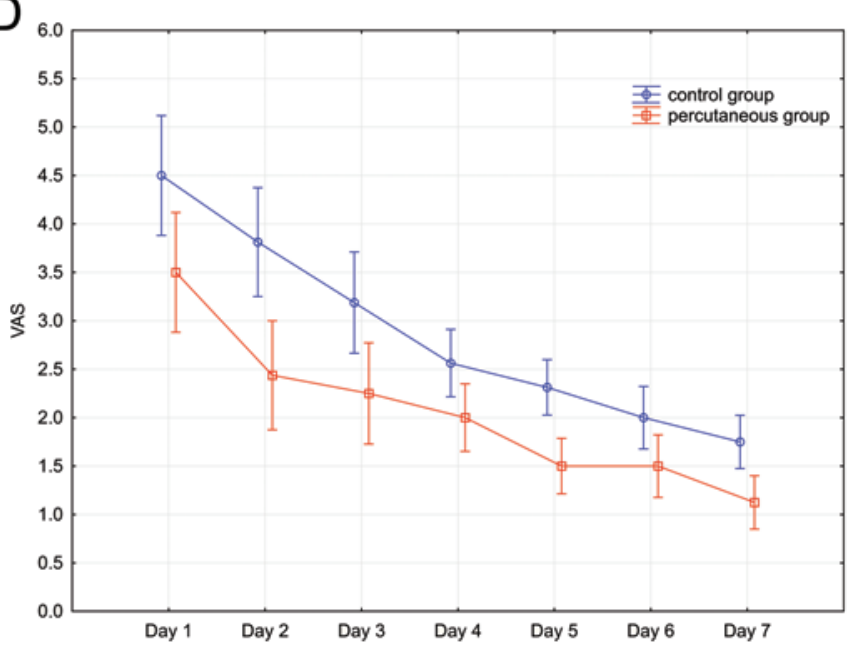

FIG. 3. Line graphs showing the change in VBI values (A), VBA angles (B), and bisegmental Cobb angles (C) over the entire study period, and the pain profile (VAS scores; D) during the first 7 days after surgery.

the potential influence of the relatively low numbers of enrolled patients on the statistical calculations, and the fact that the average age was approximately 6 years older in the control group.

There is insufficient scientific evidence within the literature concerning the feasibility and capability of percutaneous transpedicular screw systems in the field of thoracolumbar spine trauma. This rather small series is not sufficiently powered to entirely resolve this issue, but the assumption for A3 fractures (according to AO/Magerl classification) has been proven. Nevertheless, further well-designed randomized controlled studies are mandatory to clarify this topic.

\section{Conclusions}

According to the results presented here, we can confirm that the percutaneous transpedicular screw technique represents a viable option in the treatment of preselected thoracolumbar fractures. This technique can provide safe and effective treatment for A3-type AO/Magerl fractures. A significant reduction in blood loss, postoperative pain, and surgical duration are the main advantages associated with this minimally invasive technique. Clinical, functional, and radiological results are at least the same as those achieved in the open technique after a 2-year follow-up. These results do not support the traditional

TABLE 3: Overall satisfaction after 2 years of follow-up

\begin{tabular}{lcc}
\hline \multicolumn{1}{c}{ Overall Satisfaction } & Percutaneous Group & Control Group \\
\hline 1 (without any inconvenience) & 15 & 13 \\
2 (some residual difficulties) & 2 & 2 \\
3 (difficulties partially limiting common daily activities) & 1 & 2 \\
4 (significant limitation during daily activities) & 0 & 0 \\
\hline
\end{tabular}


opinion of the necessity of posterolateral fusion in such a fracture pattern.

The short-term benefits of the percutaneous transpedicular screw technique are apparent, and long-term results have to be studied in other well-designed studies to evaluate the theoretical benefit of the percutaneous technique and assess whether the results of this technique are as durable as those achieved by open surgery. The only remaining issue worth mentioning is more intensive radiation exposure to the patient and surgical team with the percutaneous technique, ${ }^{18}$ but this has not been investigated in our study.

\section{Disclosure}

The authors report no conflict of interest concerning the materials or methods used in this study or the findings specified in this paper.

Author contributions to the study and manuscript preparation include the following. Conception and design: Vanek. Acquisition of data: Vanek. Analysis and interpretation of data: Bradac, Lacman. Drafting the article: Vanek. Critically revising the article: all authors. Reviewed submitted version of manuscript: all authors. Approved the final version of the manuscript on behalf of all authors: Bradac. Statistical analysis: Bradac. Administrative/technical/material support: Konopkova, de Lacy, Lacman. Study supervision: Vanek, Benes.

\section{References}

1. Assaker R: Minimal access spinal technologies: state-of-theart, indications, and techniques. Joint Bone Spine 71:459469, 2004

2. Fan SW, Hu ZJ, Fang XQ, Zhao FD, Huang Y, Yu HJ: Comparison of paraspinal muscle injury in one-level lumbar posterior inter-body fusion: modified minimally invasive and traditional open approaches. Orthop Surg 2:194-200, 2010

3. Foley KT, Holly LT, Schwender JD: Minimally invasive lumbar fusion. Spine (Phila Pa 1976) 28 (15 Suppl):S26-S35, 2003

4. Gelalis ID, Paschos NK, Pakos EE, Politis AN, Arnaoutoglou CM, Karageorgos AC, et al: Accuracy of pedicle screw placement: a systematic review of prospective in vivo studies comparing free hand, fluoroscopy guidance and navigation techniques. Eur Spine J 21:247-255, 2012

5. Heintel TM, Berglehner A, Meffert R: Accuracy of percutaneous pedicle screws for thoracic and lumbar spine fractures: a prospective trial. Eur Spine J 22:495-502, 2013

6. Jiang XZ, Tian W, Liu B, Li Q, Zhang GL, Hu L, et al: Comparison of a paraspinal approach with a percutaneous approach in the treatment of thoracolumbar burst fractures with posterior ligamentous complex injury: a prospective randomized controlled trial. J Int Med Res 40:1343-1356, 2012

7. Kawaguchi Y, Yabuki S, Styf J, Olmarker K, Rydevik B, Matsui $\mathrm{H}$, et al: Back muscle injury after posterior lumbar spine surgery. Topographic evaluation of intramuscular pressure and blood flow in the porcine back muscle during surgery. Spine (Phila Pa 1976) 21:2683-2688, 1996

8. Khoo LT, Palmer S, Laich DT, Fessler RG: Minimally invasive percutaneous posterior lumbar interbody fusion. Neurosurgery 51 (5 Suppl):S2-166-S2-181, 2002

9. Kim KH, Kuh SU, Park JY, Kim KS, Chin DK, Cho YE: What is the importance of "halo" phenomenon around bone cement following vertebral augmentation for osteoporotic compression fracture? Osteoporos Int 23:2559-2565, 2012

10. Magerl F, Aebi M, Gertzbein SD, Harms J, Nazarian S: A comprehensive classification of thoracic and lumbar injuries. Eur Spine J 3:184-201, 1994

11. Mayer TG, Vanharanta H, Gatchel RJ, Mooney V, Barnes D, Judge L, et al: Comparison of CT scan muscle measurements and isokinetic trunk strength in postoperative patients. Spine (Phila Pa 1976) 14:33-36, 1989

12. McLain RF, Sparling E, Benson DR: Early failure of shortsegment pedicle instrumentation for thoracolumbar fractures. A preliminary report. J Bone Joint Surg Am 75:162-167, 1993

13. Rahamimov N, Mulla H, Shani A, Freiman S: Percutaneous augmented instrumentation of unstable thoracolumbar burst fractures. Eur Spine J 21:850-854, 2012

14. Rampersaud YR, Annand N, Dekutoski MB: Use of minimally invasive surgical techniques in the management of thoracolumbar trauma: current concepts. Spine (Phila Pa 1976) 31 (11 Suppl):S96-S102, S104, 2006

15. Rechtine GR, Bono PL, Cahill D, Bolesta MJ, Chrin AM: Postoperative wound infection after instrumentation of thoracic and lumbar fractures. J Orthop Trauma 15:566-569, 2001

16. Rodríguez-Vela J, Lobo-Escolar A, Joven-Aliaga E, Herrera A, Vicente J, Suñén E, et al: Perioperative and short-term advantages of mini-open approach for lumbar spinal fusion. Eur Spine J 18:1194-1201, 2009

17. Scott J, Huskisson EC: Graphic representation of pain. Pain 2:175-184, 1976

18. Smith JS, Ogden AT, Fessler RG: Minimally invasive posterior thoracic fusion. Neurosurg Focus 25(2):E9, 2008

19. Verlaan JJ, Diekerhof CH, Buskens E, van der Tweel I, Verbout AJ, Dhert WJ, et al: Surgical treatment of traumatic fractures of the thoracic and lumbar spine: a systematic review of the literature on techniques, complications, and outcome. Spine (Phila Pa 1976) 29:803-814, 2004

20. Wang HW, Li CQ, Zhou Y, Zhang ZF, Wang J, Chu TW: Percutaneous pedicle screw fixation through the pedicle of fractured vertebra in the treatment of type A thoracolumbar fractures using Sextant system: an analysis of 38 cases. Chin J Traumatol 13:137-145, 2010

21. Wild MH, Glees M, Plieschnegger C, Wenda K: Five-year follow-up examination after purely minimally invasive posterior stabilization of thoracolumbar fractures: a comparison of minimally invasive percutaneously and conventionally open treated patients. Arch Orthop Trauma Surg 127:335-343, 2007

22. Yang H, Shi JH, Ebraheim M, Liu X, Konrad J, Husain I, et al: Outcome of thoracolumbar burst fractures treated with indirect reduction and fixation without fusion. Eur Spine J 20: 380-386, 2011

23. Zdeblick TA, Sasso RC, Vaccaro AR, Chapman JR, Harris MB: Surgical treatment of thoracolumbar fractures. Instr Course Lect 58:639-644, 2009

Manuscript submitted May 22, 2013.

Accepted November 7, 2013.

Please include this information when citing this paper: published online December 20, 2013; DOI: 10.3171/2013.11.SPINE13479.

Address correspondence to: Ondrej Bradac, M.D., M.Sc., Department of Neurosurgery, Charles University and Military University Hospital Prague, U Vojenske Nemocnice 1200, Prague 6, 16902 Czech Republic. email: ondrej.bradac@uvn.cz. 